\title{
A Reduced Interval of Chromosome 9p21 Locus is Associated with Ischemic Stroke in Chinese Northern Han Population
}

\author{
Shuo Li ${ }^{1,5}$, Yu-Ming Xu ${ }^{5}$, Hong Zheng ${ }^{6}$, Edward Randell ${ }^{1}$, Hai-Zheng Wang ${ }^{5}$, Jianxun Cui ${ }^{1}$, \\ Guang Sun ${ }^{4}$, Guangju Zhai ${ }^{2}$, Fei-Yu Han ${ }^{1,2}$, Ya-Gang Xie ${ }^{1,2,3,4, *}$ \\ ${ }^{1}$ Disciplines of Laboratory Medicine, Faculty of Medicine, Memorial University of Newfoundland, St. John's, Canada \\ ${ }^{2}$ Disciplines of Genetics, Faculty of Medicine, Memorial University of Newfoundland, St. John's, Canada \\ ${ }^{3}$ Disciplines of Pediatrics, Faculty of Medicine, Memorial University of Newfoundland, St. John's, Canada \\ ${ }^{4}$ Disciplines of Medicine, Faculty of Medicine, Memorial University of Newfoundland, St. John's, Canada \\ ${ }^{5}$ Department of Neurology, First Affiliated Hospital, Zhengzhou University, Zhengzhou, P. R. China \\ ${ }^{6}$ Department of Genetics, Faculty of Medicine, Zhengzhou University, Zhengzhou, P. R. China
}

Email address:

yxie@mun.ca (Ya-Gang Xie)

${ }^{*}$ Corresponding author

\section{To cite this article:}

Shuo Li, Yu-Ming Xu, Hong Zheng, Edward Randell, Hai-Zheng Wang, Jianxun Cui, Guang Sun, Guangju Zhai, Fei-Yu Han, Ya-Gang Xie. A Reduced Interval of Chromosome 9p21 Locus is Associated with Ischemic Stroke in Chinese Northern Han Population. International Journal of Genetics and Genomics. Vol. 5, No. 1, 2017, pp. 14-18. doi: 10.11648/j.ijgg.20170501.12

Received: February 9, 2017; Accepted: March 1, 2017; Published: March 10, 2017

\begin{abstract}
The 9p21 locus, a strong risk locus for coronary arterial disease, has been also associated with other cardiovascular disease including ischemic stroke (IS) in Caucasians. However, the association between 9p21 locus and IS in Chinese Han population is still debatable because of ambiguous results reported previously. Genetic heterogeneity between Southern and Northern Chinese Han populations could be one of the reasons for this uncertainty. Four genetic variants selected from the three conjunctional LD blocks within the $44 \mathrm{~kb}$ candidate region on chromosome 9p21 were genotyped in 1,429 IS patients and 1,191 healthy controls from the Northern Chinese Han population. Among the four studied variants, the G allele of the SNP rs2383207 was significantly associated with IS with allele frequency $66.8 \%$ in patients and $63.4 \%$ in controls. This association appears to be dominant with an OR of $1.417(\mathrm{p}=0.003)$ for people with either GG or AG genotypes. We did not find any association for the other three SNPs (rs1333049, rs10757274, and rs10116277). Based our results, we conclude that the 9p21 locus is a susceptibility locus for IS in the Northern Chinese Han population; and the core risk region for IS is within an interval of less than $28 \mathrm{~kb}$.
\end{abstract}

Keywords: 9p21 Locus, Ischemic Stroke, Chinese, Genetic Association Study

\section{Introduction}

Stroke is the second most common cause of adult death worldwide, and accounts for approximately $10 \%$ of total deaths worldwide [1-3] Two-thirds of stroke-related deaths occur in developing countries, and approximately $40 \%$ of these occur within China [4-6]. Common variants in the region on chromosome 9p21 have recently been associated with increased risk for coronary arterial disease (CAD) by a number of parallel genome-wide association studies (GWAS) in Caucasians [7-10], and this result was further confirmed by several independent case control studies in Caucasians [11]. Replication studies have been successful in Japanese, Korean and Chinese Han populations [12-14, 15] although genetic heterogeneity has been reported in blacks [9-10] [15 16]. From these studies, the interval of core risk region at 9 p21 locus has been defined within a $44 \mathrm{~kb}$ LD block in Caucasians [11]. Based on the hypothesis of shared 
pathogenic mechanisms for vascular disease, studies of the 9 p21 locus have been quickly extended to a number of other cardiovascular diseases, and significant association has been reported for IS, aortic aneurysms [17], arterial stiffness [18], and aneurysmal subarachnoid haemorrhage [19]. Data from all of these studies indicate a possible atherosclerosis susceptibility of the $9 \mathrm{p} 21$ locus.

The Chinese Han population is the largest ethnic group in China which represents approximately $92 \%$ of the total population in China. Geographical variation in stroke prevalence has been found between South and North Han Chinese [20-24]. Moreover, genetic heterogeneity has also been reported between Southern and Northern Chinese Han populations [25-28].

The association between certain genetic variants within the 9p21 locus and CAD has been confirmed [14] in the Chinese Han population. However, the association between the 9p21 locus and IS in the Chinese Han population remains debatable because of inconsistent results from the different studies. While, insufficient sample size can be a factor, potential effects of genetic heterogeneity between Southern and Northern Chinese Han populations, as described in previous studies, may also be a factor confounding results [14] [29]. Therefore, continued efforts with increased patient numbers and targeting of specific sub Han populations are necessary to define the association between the $9 \mathrm{p} 21$ locus and IS in Chinese Han individuals. In the present study, genotyping of four selected genetic variants from three conjunctional LD blocks within the $44 \mathrm{~kb}$ candidate region on chromosome 9p21 were performed in 1,429 ischemic stroke (IS) patients, and 1,191 normal controls from the Northern Han Chinese population.

\section{Materials and Methods}

\subsection{Patients and Controls}

1,429 consecutive IS patients were recruited by 18 hospitals within Henan province during the period from February 2006 to March 2007. Clinical diagnoses of IS patients were based on the WHO criteria for IS (1998) plus evidence from MRI or CT exam on ischemic lesions corresponding to the neurological deficits. The 1,191 normal controls were selected from ethnically and geographically matched individuals without history of myocardial infarction or stroke who presented in hospitals for routine health examinations. Ethics approval of the present study was granted by the Human Investigations Committee of Memorial University and Ethics Boards of Zhengzhou University.

\subsection{SNP Genotyping}

Genomic DNA was isolated from peripheral blood collected from patients and controls using standard methods [30]. A chromosomal region between position rs1333049 and the rs10116277 was targeted using HapMap CHB (Chinese
Han Beijing). Four SNPs including rs1333049, rs2383207, rs10757274, and rs10116277 were selected from the $44 \mathrm{~kb}$ LD block in the region of 9 p21.9 to 9 p22.1 (NCBI build 36.2, HapMap database for the CEU population) which have previously been associated with MI and IS in Caucasians. Genotyping was conducted using Taq Man SNP genotyping technology on real-time PCR (ABI Prism ${ }^{\circledR} 7000$ Sequence Detection System).

\subsection{Statistical Analysis}

Hardy-Weinberg equilibrium (HWE) was tested for each of the four SNPs using the exact Chi-square test, and none of them was out of HWE. Logistic regression modeling implemented in SPSS v16.0 (SPSS Inc.) was used to test the association between each of the SNPs and the IS. Odds ratios (OR) were calculated as a measure of the relative risk for stroke and were given with $95 \%$ CIs. A $\mathrm{P}<0.05$ (two-tailed) was considered statistically significant. Statistical power was calculated using QUANTO V1.2.3 software. Linkage disequilibrium between the two analyzed variants was calculated as $\mathrm{D}^{\prime}$, which ranges from 0 (no linkage disequilibrium) to 1 or -1 (complete linkage disequilibrium). The software QUANTO version 1.2.3 was used for the calculation of statistical power.

\section{Results}

In the present study, the patients group consist of $60.7 \%$ $(\mathrm{n}=868)$ males and $39.3 \%$ females $(\mathrm{n}=561)$ compared with $63.5 \%$ males $(n=756)$ and $36.5 \%$ females $(n=435)$ in the control group. The mean $\pm \mathrm{SD}$ age in patient and control groups are: $62.7 \pm 11.8$ and $58.5 \pm 9.2$, respectively.

The minor allele frequencies (MAF) of the four tested SNPs ranged from 0.31 to 0.49 , according to HapMap CHB (Chinese Han Beijing) data, with a mean MAF of 0.3975 for the four SNPs. Given the disease prevalence at $0.5 \%, 1429$ patients and 1197 controls yielded a statistical power $>0.8$ to detect an OR of 1.50 for the MAF of 0.40 for the tested SNPs at a significance level of 0.05 (two-tailed) under recessive, dominant and additive models.

The four selected SNPs were genotyped in 1,429 IS patients and 1,191 controls. The genotype distributions, allelic frequencies of each studied genetic variants in patients and controls are given in Table 1. The genotype frequencies of the four studied genetic variants in the control population were all under the Hardy-Weinberg equilibrium (all $\mathrm{P}>0.05$ ). In the association analysis, only the common allele $(\mathrm{G})$ of SNP rs2383207 was significantly associated with increased risk for IS with an $\mathrm{OR}=1.162$ for $\mathrm{G}$ allele $(\mathrm{P}$ - value $=0.01$ ), and $\mathrm{OR}=1.417$ for $\mathrm{AG}+\mathrm{GG}$ genotypes $(\mathrm{P}-\mathrm{value}=0.003$. This association was observed in both heterozygous AG $(\mathrm{OR}=1.382, \mathrm{P}=0.010)$ and homozygous $\mathrm{GG} \quad(\mathrm{OR}=1.417$, $\mathrm{P}=0.003$ ) statuses. 
Table 1. Genotype distributions and allelic frequencies of rs10116277, rs 10757274, rs2383207, and rs1333049 in IS patients and controls.

\begin{tabular}{|c|c|c|c|c|}
\hline Genotype & Stroke $(n=1,429)$ & $\mathrm{NC}(\mathrm{n}=1,191)$ & OR $(95 \% \mathrm{CI})$ & P-value \\
\hline \multicolumn{5}{|l|}{ Rs10116277 } \\
\hline GG & $137(9.6 \%)$ & $137(11.5 \%)$ & & \\
\hline GT & $606(42.4 \%)$ & $501(42.1 \%)$ & $1.210(0.928-1.576)$ & 0.159 \\
\hline TT & $686(48.0 \%)$ & $553(46.4 \%)$ & $1.241(0.955-1.612)$ & 0.106 \\
\hline \multicolumn{5}{|l|}{ Rs10757274 } \\
\hline AA & $453(31.7 \%)$ & $354(29.7 \%)$ & & \\
\hline AG & $676(47.3 \%)$ & $600(50.4 \%)$ & $0.880(0.737-1.051)$ & 0.159 \\
\hline GG & $300(21.0 \%)$ & $237(19.9 \%)$ & $0.989(0.794-1.232)$ & 0.923 \\
\hline \multicolumn{5}{|l|}{ Rs2383207 } \\
\hline AA & $154(10.8 \%)$ & $174(14.6 \%)$ & & \\
\hline $\mathrm{AG}$ & $642(44.9 \%)$ & $525(44.1 \%)$ & $1.382(1.081-1.766)$ & 0.010 \\
\hline GG & $633(44.3 \%)$ & $492(41.3 \%)$ & $1.454(1.136-1.861)$ & 0.003 \\
\hline \multicolumn{5}{|l|}{ Rs1333049 } \\
\hline GG & $381(26.7 \%)$ & $334(28.0 \%)$ & & \\
\hline $\mathrm{GC}$ & $733(51.3 \%)$ & $571(47.9 \%)$ & $1.125(0.937-1.352)$ & 0.206 \\
\hline $\mathrm{CC}$ & $315(22.0 \%)$ & $286(24.0 \%)$ & $0.966(0.777-1.200)$ & 0.752 \\
\hline
\end{tabular}

Data from logistic regression analysis on rs2383207 showed that heterozygous AG (OR: $1.359,95 \% \mathrm{CI}: 1.057$ to $1.745, \mathrm{P}=0.017)$ and homozygous $\mathrm{GG}(\mathrm{OR}: 1.408,95 \% \mathrm{CI}$ : 1.095 to $1.811, \mathrm{P}=0.008)$ are associated with $\mathrm{IS}$ after adjustment with age and sex. The details of the regression analysis are given in Table 2.

Table 2. Multivariable analysis of the association between SNP rs2383207 and $I S^{*}$.

\begin{tabular}{llll}
\hline & OR & 95\%CI & P \\
\hline Age ( \pm SD: $62.7 \pm 11.8)$ & 1.037 & $1.030-1.045$ & $<0.001$ \\
Sex (M vs F) & 1.164 & $0.990-1.368$ & 0.066 \\
CC & - & - & - \\
CG & 1.359 & $1.057-1.745$ & 0.017 \\
GG & 1.408 & $1.095-1.811$ & 0.008 \\
\hline
\end{tabular}

*the analysis was done with logistic regression modeling. OR - Odds Ratio. Genotype CC was reference.

The results of non-association in the other three studied SNPs indicate that the interval of the candidate region for IS on 9p21 locus could be less than $28 \mathrm{~kb}$.

\section{Discussion}

The GWAS has become a powerful tool in the identification of multiple previously unknown genetic susceptibility factors involved in complex disease. The traditional candidate-gene association study is, however, still a useful method for replication studies of the results from GWAS. In the present study, four genetic variants (rs1333049, rs2383207, rs10757274 and rs10116277) selected from the $44 \mathrm{~kb}$ core candidate region for CAD and IS were studied by genotyping 1,429 IS patients and 1,191 healthy controls from the Chinese Han population. This study successfully associated the 9p21 locus with IS in the Chinese Han population.

Among the four studied SNPs only the rs2383207 was associated with increased risk for IS, although, all of four SNPs are within the $44 \mathrm{~kb}$ core candidate region for CAD. This result may suggest a smaller LD block encompassing rs2383207 $(28 \mathrm{~kb})$ in the Chinese Han population, compared with Caucasians (44kb). According to the data from HapMap CHB (Chinese Han Beijing), the rs10757274 and the rs2383207 are within the same $28 \mathrm{~kb}$ LD block $\left(\mathrm{r}^{2}>0.8\right)$ in the Chinese population, and the rs1333049 and the rs10116277 are scattered into two flanking LD blocks in Han Chinese. Negative association of rs10757274 may indicate that the interval for the core candidate region for IS may be restricted to a region that is smaller than $28 \mathrm{~KB}$. Focusing study on this narrowed core risk region for IS could help to further characterize and define the DNA sequence that is pathogenically responsible for cardiovascular diseases, especially for IS. A further sequence characterization in this smaller interval of the core risk region of 9 p21 is underway.

Our results are consistent with those of $\mathrm{Hu}$ et al (2009) [28] in showing an association between genetic variants within the 9p21 locus and IS in the Chinese Han population. Of interest, the association in the $\mathrm{Hu}$ et al study was only obtained by haplotype analysis, but genotype analysis failed to show a significant association. This failure to show significant association by genotype analysis by $\mathrm{Hu}$ et al $(2009)^{28}$ may be due to possible genetic heterogeneity between the Southern and Northern Han populations. It is possible that the association between IS and variants within the 9p21 locus are stronger in Northern Han Chinese due to different genetic modifiers. The Chinese Han population can be generally divided into two sub-populations, southern and northern Han. Genetic heterogeneity has been reported between these two sub-populations [24-27]. In the $\mathrm{Hu}$ et al study, the studied subjects were collected from Hubei province which is geographically belonging to South China. While, the subjects in the present study were collected from Henan Province which geographically belongs to North China. The minor differences between these two studies further support the genetic heterogeneity between Northern and Southern Han populations.

In the present study, the rs2383207 $\mathrm{G}$ allele was significantly associated with increased risk for IS $\left(\mathrm{OR}=1.382 ; \mathrm{P}_{\text {heterozygot }}\right.$ value $=0.01$ and $\mathrm{OR}=1.454 ; \mathrm{P}_{\text {homzygot }}$ value $=0.003$ ). These results suggest an additive effect of the rs $2383207 \mathrm{G}$ allele in the genetic susceptibility for IS. 
As the effect on CAD risk at the $9 \mathrm{p} 21$ locus has been found to be independent of known risk factors, including hypertension, diabetes, hyperlipidemia, and obesity, we therefore did not integrate these data in our result analysis. The average age of patients in our study was 4.2 years older than in the control group which could lead to under estimation of risk based on the studied genetic variants. This challenge will be minimized in our further sample collection by focusing on controls subjects of older age.

\section{Conclusion}

In this study, the four genetic variants selected from the three conjunctional LD blocks within the $44 \mathrm{~kb}$ candidate region on chromosome 9p21 were genotyped in 1,429 IS patients and 1,191 healthy controls from the Northern Chinese Han population. The SNP rs2383207 was significantly associated with IS. The results of nonassociation in the other three studied SNPs indicate that the interval of the candidate region for IS on 9 p21 locus could be less than $28 \mathrm{~kb}$. We, therefore, suggest that the 9p21 locus is a susceptibility locus for IS in the Northern Chinese Han population; and the core risk region for IS is within an interval of less than $28 \mathrm{~kb}$.

\section{Acknowledgments}

This research was supported by. A. R. Cox Research Grant of Memorial University, Health Care Foundation of Eastern Health.

\section{Reference}

[1] Murray CJL, Lopez AD (1997) Mortality by cause for eight regions of the world: global burden of disease study. Lancet 349: $1269-1276$.

[2] Bonita R, Mendis S, Truelsen T, et al (2004) The global stroke initiative. Lancet Neurology 3: 391-393.

[3] Donnan GA, Fisher M, Macleod M, et al (2008) Stroke. Lancet 371: 1612-1623.

[4] Cheng YC, Stanne TM, Giese AK, Ho WK, et al. (2016) Genome-wide association analysis of young-onset stroke identifies a locus on chromosome 10q25 near habp2. Stroke; $a$ journal of cerebral circulation. 2016; 47: 307-316.

[5] Feigin VL, Lawes CM, Bennett DA, et al (2003) Stroke epidemiology: a review of population-based studies of incidence, prevalence, and case-fatality in the late 20th century. Lancet Neurology 2: 43-53.

[6] Reddy KS, Yusuf S. (1998) Emerging epidemic of cardiovascular disease in developing countries. Circulation 97: 596-601.

[7] The Wellcome Trust Case Control Consortium 2007.

[8] Helgadottir A, Thorleifsson G, Manolescu A, et al (2007) Common variant on chromosome 9p21 affects the risk of myocardial infarction. Science. 8;316 (5830): 1491-3.
[9] McPherson R, Pertsemlidis A, Kavaslar N, et al. (2007) A Common allele on chromosome 9 associated with coronary heart disease. Science 316: 1488-1491.

[10] Samani NJ, Erdmann J, Hall AS, et al. (2007) Genome wide association analysis of coronary artery disease. N Engl J Med. 357: 443-453.

[11] Matarin M, Brown WM, Singleton A, Hardy JA, Meschia JF. Whole genome analyses suggest ischemic stroke and heart disease share an association with polymorphisms on chromosome 9p21. Stroke 2008; 39: 1586-1589.

[12] Shen GQ, Li L, Rao S, Abdullah KG, et al. (2008) Four SNPs on chromosome 9p21 in a South Korean population implicate a genetic locus that confers high cross-race risk for development of coronary artery disease. Arterioscler Thromb Vasc Biol. 28: 360-365.

[13] Hinohara K, Nakajima T, Takahashi M, et al. (2008) Replication of the association between a chromosome 9p21 polymorphism and coronary artery disease in Japanese and Korean populations. J Hum Genet 53: 357-359.

[14] Ding H, Xu Y, Wang XJ, et al (2009) 9p21 is a Shared Susceptibility Locus Strongly for Coronary Artery Disease and Weakly for Ischemic Stroke in Chinese Han Population. Circ Cardiovasc Genet 2; 338-346.

[15] Bi J, Yang L, Liu D, Wu J, Tong X, Cen S, Zhou D, Zhang T, Yi L. Sequence variants on chromosome 9p21 are associated with ischemic stroke and the lipids level in chinese han population. (2015) Journal of stroke and cerebrovascular diseases: the official journal of National Stroke Association 24: 894-900.

[16] Schunkert H, Gotz A, Braund P, et al. (2008) Repeated replication and a prospective meta-analysis of the association between chromosome 9p21.3 and coronary artery disease. Circulation117: 1675-1684.

[17] Helgadottir A, Thorleifsson G, Magnusson KP, et al. (2008) The same sequence variant on $9 \mathrm{p} 21$ associates with myocardial infarction, abdominal aortic aneurysm and intracranial aneurysm. Nature Genetics 40: 217-224.

[18] Bjorck HM, Lanne T, Alehagen U, et al. (2009) Association of genetic variation onchromosome 9 p21.3 and arterial stiffness. J Intern Med 265: 373e81.

[19] Olsson S, Csajbok L, Jood K, et al. (2011) Association between genetic variation on chromosome 9p21 and aneurismal subarachnoid haemorrhage. J Neurol Neurosurg Psychiatry 82: 384 e388.

[20] Wu ZS, Yao CH, Zhao D, et al (2001) Sino-MONICA project: A collaborative study on trends and determinants in cardiovascular diseases in China, part I: morbidity and mortality monitoring. Circulation 103: 462-468.

[21] Li SC, Schoenberg BS, Wang CC, et al. (1985) Cerebrovascular disease in the People's Republic of China: epidemiologic and clinical features. Neurology 35: 1708-1713.

[22] Chen XM. Xinde W, Mingxun T, et al. Epidemiology of cerebrovascular diseases. Cerebrovascular Diseases. Chinese Science and Technology Publishing House 1993.

[23] Wang CC, Cheng XM, Li SC. (1985) Epidemiological survey of neurological disorders in six urban areas of People's Republic of China. Chinese Neurosurgery Journal 1: 2-7. 
[24] Cheng XM, Ziegler DK, Lai YC, et al. (1995) Stroke in China, 1986 through 1990. Stroke 26: 1990-1994.

[25] Wen B, Li H, Lu DR, et al. (2004) Genetic evidence supports demic diffusion of Han culture. Nature 431: 302-305.

[26] Xu SH, Yin XY, Li SL, et al. (2009) An Y, and et al. Genomic Dissection of Population Substructure of Han Chinese and Its Implication in Association Studies. The American Journal of Human Genetics 85: 762-774.

[27] Chen JM, Zheng HF, Bei JX, et al. (2009) Genetic Structure of the Han Chinese Population Revealed by Genome-wide SNP Variation. The American Journal of Human Genetics 85: 775785.
[28] Yue X, Tian L, Fan X, Xu G, Shi FD, Liu X. (2015) Chromosome 9p21.3 variants are associated with cerebral infarction in chinese population. Journal of molecular neuroscience: $M N .2015$; 56: 546-552.

[29] $\mathrm{Hu}$ WL, Li SJ, Liu DT, et al. (2009) Genetic variants on chromosome 9p21 and ischemic stroke in Chinese. Brain Research Bulletin 79: 431-435.

[30] Miller SA, Dykes DD, Polesky HF. (1998) A simple salting out procedure for extracting DNA from human nucleated cells. Nucleic Acids Res. 16: 1215. 